\title{
Wertigkeit von pathologisch-anatomischen und staubanalytischen Unter- suchungen bei asbestassoziierten Berufskrankheiten am Beispiel der BK 4104
}

\author{
Relevance of Pathological Examinations and Lung Dust Analyses in the Context of Asbestos-Associated Lung \\ Cancer-No. 4104 of the List of Occupational Diseases in Germany
}

Autoren

Institute
V. Neumann ${ }^{1}$, T. Kraus ${ }^{3}$, M. Fischer ${ }^{1}$, S. Löseke ${ }^{1}$, A. Tannapfel ${ }^{1,2}$

Deutsches Mesotheliomregister am Institut für Pathologie der Ruhr-Universität Bochum am Berufsgenossenschaftlichen Universitätsklinikum Bergmannsheil

Institut für Pathologie der Ruhr-Universität Bochum am Berufsgenossenschaftlichen Universitätsklinikum Bergmannsheil

${ }^{3}$ Institut für Hygiene and Umweltmedizin am Universitätsklinikum Aachen eingereicht 30.6.2009

akzeptiert $\quad 4.8 .2009$

Bibliografie

DOI $10.1055 / \mathrm{s}-0029-1215093$

Online-Publikation: 18. 9. 2009

Pneumologie 2009; 63:

588-593 @ Georg Thieme

Verlag KG Stuttgart · New York ISSN 0934-8387

Korrespondenzadresse Dipl.-Biol. Volker Neumann

Deutsches Mesotheliomregister am Institut für Pathologie Bürkle-de-la-Camp-Platz 1 44789 Bochum

Volker.Neumann@

ruhr-uni-bochum.de

\section{Zusammenfassung}

\section{$\nabla$}

Diese Arbeit diskutiert die Relevanz pathologischanatomischer und lungenstaubanalytischer Untersuchungen im Rahmen der versicherungsmedizinischen Bewertung von asbestassoziierten Lungenkarzinomen anhand von drei Fallbeispielen. Die Fallbeispiele 1 und 2 zeigen, dass konventionelle computertomografische Untersuchungen mit einer Auflösungsgrenze von $3 \mathrm{~mm}$ nicht immer den Nachweis asbestassoziierter Pleuraveränderungen führen können. Erst im Rahmen der Obduktionen wurden Pleuraplaques gesichert und damit waren die Kriterien für eine Berufskrankheit nach Ziffer 4104 der Berufskrankheitenverordnung erfüllt. Das Fallbeispiel 3 macht deutlich, dass bei pathologisch-anatomischen Routine-Untersuchungen - ohne Kenntnis einer zur Diskussion stehenden asbestassoziierten Erkrankung - die Diagnose einer Minimalasbestose deutlich erschwert ist. Erst gezielte histologische Untersuchungen (400-fache Vergrößerung, Eisenfärbung) und die Zuhilfenahme der Ergebnisse der staubanalytischen Untersuchung ermöglichten den Nachweis einer Minimalasbestose. Eine pathologisch-anatomische Untersuchung unter Berücksichtigung der Lungenstaubanalytik ist wie gezeigt - bei der Bewertung asbestassoziierter Lungenerkrankungen von hoher Wertigkeit. Die Nichtausschöpfung aller Beweismittel kann dazu führen, dass entsprechende Berufskrankheiten nicht entschädigt werden. Pathologisch-anatomische Untersuchungen sind und bleiben somit unverzichtbar. Im Falle einer Verfügbarkeit von ausreichendem Lungengewebe ist die Ablehnung einer Berufskrankheit nach Ziffer 4104 ohne diese Untersuchungen daher nicht gerechtfertigt.

\section{Abstract \\ $\nabla$}

This report discusses the relevance of pathological-anatomical examinations and lung dust analyses in the context of asbestos-related lung cancer on the basis of three case reports. The cases one and two demonstrate a limited performance of conventional computed tomography scanning with a resolution of $3 \mathrm{~mm}$ for the detection of asbestos-related pleural diseases. In these cases, only the autopsy was able to confirm the diagnosis of pleural plaques and, therefore, the German criteria for occupational disease No. 4104 of the list of the occupational diseases were fulfilled. Case three clearly shows that routine pathological examinations, especially without the consideration of an occupational disease, could not always successfully obtain the diagnosis of a grade I asbestosis. Only intense histological examinations (iron-staining, $400 \times$ magnification) in combination with lung dust analysis were able to provide such a diagnosis. As shown here, pathologicalanatomic examinations including lung dust analysis are highly valuable for the estimation of asbestos-related lung diseases. A merely partial consideration of all possible evidence forms can be responsible for the rejection of a reasonable compensation claim for an occupational disease. Therefore pathological-anatomic examinations are indispensable today and in the future. A definite rejection of occupational disease No. 4104 without an analysis of lung parenchyma is not justified. 


\section{Einleitung}

\section{$\nabla$}

Im Rahmen von Berufskrankheitenverfahren zur Fragestellung asbestassoziierter Lungenkarzinome kommt der Sicherung der vom Gesetzgeber benannten Brückenbefunde eine entscheidende Bedeutung zu.

Die Kriterien für eine Berufskrankheit (BK) nach Ziffer 4104 der Berufskrankheitenverordnung (BKVO) sind erfüllt, wenn ein Lungenkarzinom vollbeweislich gesichert ist, eine unfallversicherte Asbestexposition (Vollbeweis) vorliegt und ggf. ein Brückenbefund [1] nachgewiesen werden kann. Als Brückenbefunde für eine BK nach Ziffer 4104 (asbestassoziierter Lungenkrebs oder Kehlkopfkrebs) werden aufgeführt:

- eine Asbeststaublungenerkrankung (im Sinne der BK 4103)

- eine durch Asbeststaub verursachte Erkrankung der Pleura (im Sinne der BK 4103)

- und die sogenannte technische Brücke mit Nachweis einer kumulativen Asbestfaserstaubdosis am Arbeitsplatz von mindestens 25 Faserjahren $\left(25 \times 10^{6}\left[\left(\right.\right.\right.$ Fasern $\left./ \mathrm{m}^{3}\right) \times$ Jahre] $)$.

Nach den Erfahrungen des Deutschen Mesotheliomregisters erfolgt die Abklärung eines Verdachtes auf eine BK nach Ziffer 4104 in der Regel in nachstehender Abfolge:

1. Sicherung eines primären Lungenkarzinoms unter Berücksichtigung der klinischen Befunde [2],

2. radiologische Abklärung einer Asbestose und/oder asbestassoziierter Pleuraveränderungen. Bei negativem Befund ggf. pathologisch anatomische Untersuchungen entsprechender Gewebeproben,

3. ggf. sequentiell oder zeitgleich arbeitstechnische Ermittlungen mit Quantifizierung der kumulativen Asbestfaserjahrdosis,

4. bei weniger als 25 Asbestfaserjahren und fehlenden asbestassoziierten Pleuraveränderungen Prüfung einer Minimalasbestose.

Die o.g. Ermittlungen laufen im Idealfall parallel, häufig aber sukzessive. Dabei kann es z.B. vorkommen, dass die Berechnung der kumulativen Asbestfaserstaubdosis erst spät abgeschlossen werden kann und pathologisch-anatomische und lungenstaubanalytische Ergebnisse bereits vorliegen. Ziel aller beteiligten Instanzen ist es, möglichst schnell - vor allem bereits zu Lebzeiten der Versicherten - die versicherungsmedizinischen Gesichtspunkte abzuklären.

Von grundsätzlicher Bedeutung ist, dass eine Asbestose Grad I = Minimalasbestose radiologisch nicht diagnostiziert werden kann, da diese minimale asbestassoziierte Veränderung selbst mittels des hochauflösenden CT (im HRCT können Strukturen erst ab einer Dicke von 0,1 mm sichtbar gemacht werden) nicht zu erfassen ist [3-5]. Aus Sicht des Mesotheliomregisters ist festzustellen, dass es eine Tendenz gibt, bei vermeintlich niedrigen kumulativen Asbestfaserstaubdosen auf ergänzende pathologisch-anatomische und lungenstaubanalytische Untersuchungen zu verzichten. Obduktionen werden teilweise auch deshalb nicht durchgeführt werden, weil die Unfallversicherungsträger nicht immer über den Tod des Patienten informiert werden. Ferner wird unzutreffend gefolgert, dass nur auf Basis der radiologischen, klinischen Befunde sowie arbeitstechnischen Erhebungen die versicherungsmedizinischen Aspekte vollständig, auch ohne Obduktion abgeklärt werden können. So werden sogar radiologische Begutachtungen zur Fragestellung nach einer Minimalasbestose herangezogen, obwohl diese radiologisch invisibel bleibt $[4,5]$.
Die Diagnose einer Minimalasbestose erfolgt in exakter Anwendung der Kriterien der Deutschen Gesellschaft für Pathologie [6] und der so genannten Helsinki Kriterien [7]. Die Merkmale für die histologische Diagnostik einer Minimalasbestose/Asbestose wurden nochmals im Jahr 2004 von der Amerikanischen Thorax-Gesellschaft bestätigt [8]. Dabei ist es von grundlegender Bedeutung, dass es keinen staubanalytischen Grenzwert für die Minimalasbestose/Asbestose gibt [6,9].

Im Deutschen Mesotheliomregister [9] werden Lungenstaubanalysen durchgeführt, wenn

- pleurale Brückenbefunde fehlen und auch der sog. technische Brückenbefund von $\geq 25$ FJ nicht erreicht ist und das Vorliegen einer Minimalasbestose zu prüfen ist,

- fibrosierende Lungenveränderungen unterschiedlicher Genese differenzialdiagnostisch gegeneinander abzugrenzen sind,

- eine berufliche Asbestexposition nicht ausreichend identifizierbar und/oder quantifizierbar ist und eine asbestassoziierte Berufskrankheit zur Diskussion steht.

Staubanalytische Untersuchungen erfolgen an Operationspräparaten, die in der Regel unter kurativen bzw. seltener palliativen Gesichtspunkten entnommen wurden, bei Tumorleiden naturgemäß auch zu diagnostischen Zwecken. Die Proben werden in der Regel nicht unter dem primären Aspekt der Abklärung einer möglichen Berufskrankheit entnommen.

\section{Material und Methoden \\ $\nabla$}

Basis dieser Untersuchung sind die Befunde, die bei 3 Patienten mit Verdacht auf eine Berufskrankheit der Ziffer 4104 (2007 bis 2009) erhoben wurden. Diese drei Beispiele können als stellvertretend für weitere Patienten angesehen werden, es handelt sich um wiederkehrende Beobachtungen. Derartige Fallkonstellationen werden im Deutschen Mesotheliomregister nicht gesondert registriert. Grundlage der Untersuchungen waren in 2 Fällen die im Rahmen von Obduktionen gewonnenen Ergebnisse, im dritten Fall stand ein Operationspräparat zur Verfügung. Informationen zum beruflichen Werdegang, zur Exposition gegenüber Asbest und anderen Gefahrstoffen wurden den im Deutschen Mesotheliomregister vorliegenden Unterlagen entnommen. Die Sicherung der Tumordiagnose gründet sich auf histomorphologische und histochemische Untersuchungen und ergänzende immunhistochemische Reaktionen.

\section{Lungenstaubanalysen}

\section{$\nabla$}

Zur Quantifizierung der Konzentrationen der Asbestkörper (Asbestkörper pro $\mathrm{cm}^{3}$ Lungenparenchym oder g Feuchtgewebe) wurden Lungenstaubanalysen durchgeführt [10]. Soweit möglich werden bis zu vier Gewebeproben unterschiedlicher Lokalisation entnommen. Die lichtmikroskopische Filteranalyse erfolgt bei 200- bis 400-facher Vergrößerung im Differenzial-InterferenzKontrast und Polarisationsbild [10-12]. Die Asbestbelastung der Lunge pro $\mathrm{cm}^{3}$ wird verglichen mit Werten von sog. „Normallungen“ (von Personen ohne berufliche Asbestbelastung). Der Normalwert liegt bei $9 \pm 13$ Asbestkörper (schief normale Verteilung) pro $\mathrm{cm}^{3}$ Lungengewebe $[12,13]$. Die quantitative lichtmikroskopische Analyse von Lungengewebe ist der „Gold-Standard“ für die Bestimmung der Konzentration von Asbestkörpern [14]. Es wurde eine Beziehung zwischen der Anzahl der Asbestkörper in 
Schnittpräparaten (Eisenblaufärbung) und den staubanalytisch ermittelten Anzahlkonzentrationen nachgewiesen [15].

\section{Fallbeispiele}

\section{Fallbericht 1}

Männlicher Patient geboren April 1934.

Beruf: Betriebshandwerker und Tiefbauarbeiter.

Exposition: Asbestexposition:

1.7.1968 bis 31.12.1984 und 1.1.1988 bis 31.12.1990:

3,9 Asbestfaserjahre.

Polyzyklische Kohlenwasserstoffe: weniger als 1 BaP-Jahr.

Krankengeschichte: Im Mai 2006 erfolgte die Erstdiagnose eines Lungenkarzinoms (Plattenepithelkarzinom). Anschließend Einleitung einer Chemotherapie. Der Patient verstarb im Oktober 2006.

Radiologische Stellungnahme: „Auf den vorgelegten Thorax-CT Aufnahmen (vom Mai 2006) lassen sich keine typischen Plaques erkennen.“ „Radiologisch auf den Aufnahmen keine Brückenbefunde für eine BK 4104.“

Pathologie: Im Rahmen der Obduktion wurde die Diagnose eines Lungenkarzinoms bestätigt. Als Todesursache wurde das Tumorleiden festgestellt. Weiterhin zeigten Rippenfell und Zwerchfell mehrere bilaterale kostale bzw. diaphragmale typische Pleuraplaques $(7 \times 3 \times 0,3 \mathrm{~cm}$ Länge, Breite, Höhe; $\bullet$ Abb. 1$)$.

Das Lungenparenchym wies keine Asbestose (Grad II-IV) oder Minimalasbestose (Grad I) auf.

Pathologisch anatomische Bewertung: Kriterien für eine BK 4104 erfüllt.

Anschließende Arbeitsmedizinische Stellungnahme: Thorax-CT zeigt keine Pleuraplaques. „Insgesamt handelt es sich um einen interessanten Fall, wobei die Tatsache, dass die Plaques im CT nicht gesehen wurden, wohl darauf zurückzuführen ist, dass diese sehr flach waren (Dicke 0,3 $\mathrm{cm}=3 \mathrm{~mm}=$ Auslösungsgrenze des CTs)."

\section{Fallbericht 2}

Männlicher Patient geboren März 1949.

Beruf: Schlosser (u. a. im Panzerbau).

Exposition: Asbestexposition: 1.7.1973 bis 28.5.1982: Asbestfaserjahre 1,3 .

Krankengeschichte: Im Januar 2007 Diagnose eines Lungenkarzinoms (Adenokarzinom). Anschließend Therapie einer einseitigen Pleurakarzinose mittels Talkumpleurodese.

Fachradiologische Stellungnahme: 2007

Es lagen Röntgen-Thorax und CT-Aufnahmen (vom März 2007, Schichtdicke $10 \mathrm{~mm}$ ) vor. In der fachradiologischen Stellungnahme wird erklärt: „Keine eindeutigen asbestassoziierten Pleuraveränderungen. Auf einer Schicht ist zwar eine minimale Pleuraunregelmäßigkeit dorsal erkennbar, es ist jedoch anhand dieser einen Schicht nicht ausreichend zu differenzieren, ob es sich um eine plaqueartige Veränderungen oder um Abschnitte physiologischer Strukturen handelt. Keine asbestassoziierte Lungenfibrose."

Pathologie: Der Patient verstarb im März 2007, es wurde eine Obduktion durchgeführt: Sicherung der Diagnose eines Lungenkarzinoms. Todesursächlich waren die Folgen der Tumorerkrankung. Das Rippenfell wies beiderseitige hyaline Pleura- und Zwerchfellplaques auf $(\bullet$ Abb. 2).

Keine Asbestose (Grad II - IV) oder Minimalasbestose (Grad I).

Pathologisch anatomische Bewertung: Kriterien für eine BK 4104 erfüllt.

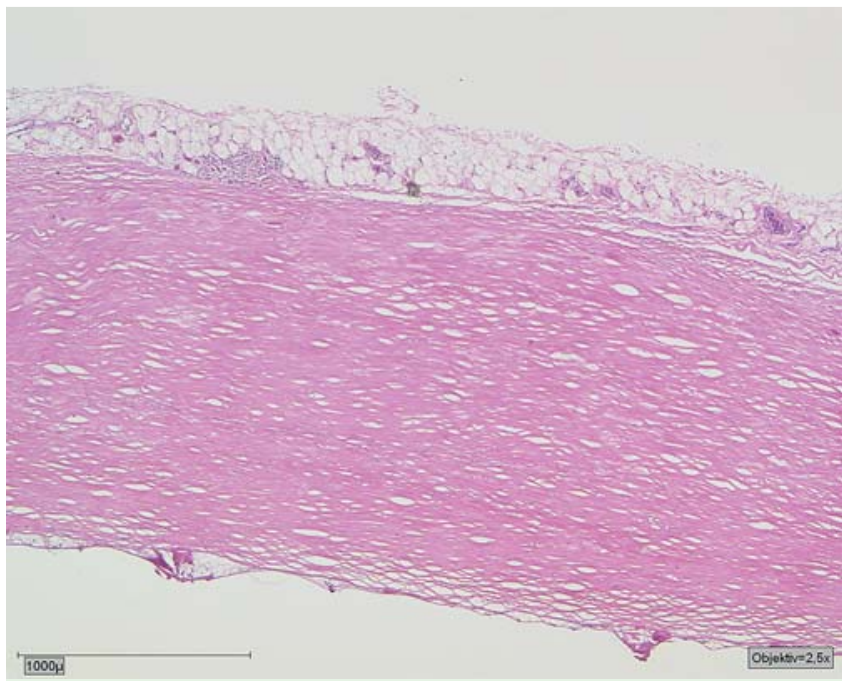

Abb. 1 Fall 1 Pleuraplaque mit einer Dicke von weniger als $3 \mathrm{~mm}$ bei $25-$ facher Vergrößerung (HE-Färbung).

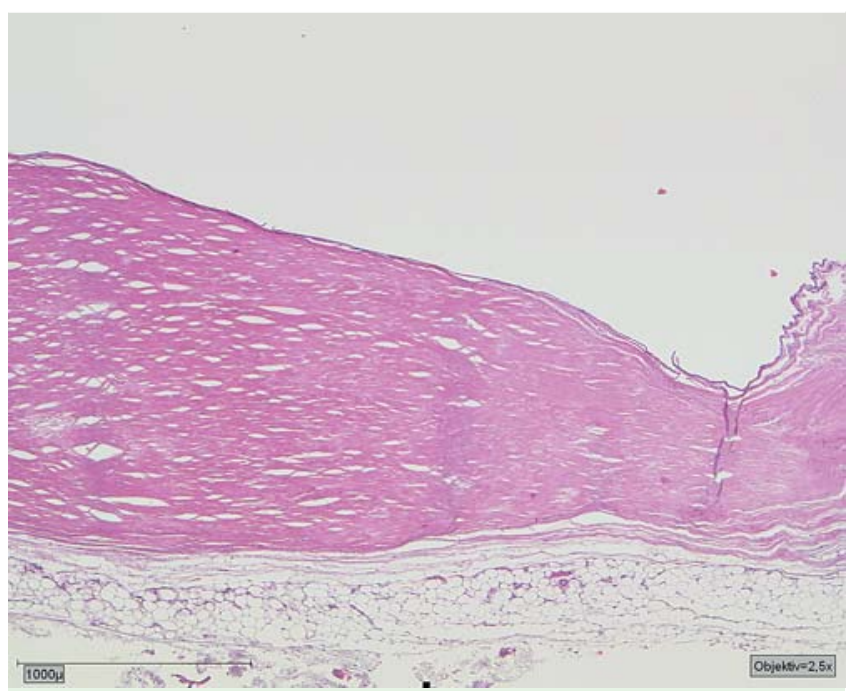

Abb. 2 Fall 2 Pleuraplaque mit deutlich ausgeprägter korbgeflechtartiger Anordnung der Kollagenfasern bei 25 -facher Vergrößerung (HE-Färbung).

\section{Fallbericht 3}

Männlicher Patient geboren Januar 1933.

Beruf: Tischler, teils mit Tätigkeiten auch im Fensterbau.

Exposition: Asbestexposition: Stellungnahme der Präventionsabteilung (Oktober 2007): „Nach dem Kenntnisstand des technischen Sachverständigen von Vergleichsarbeitsplätzen ist davon auszugehen, dass der Betroffene in der Zeit vom 01.03.1966 bis Ende 1970 in gewissem Umfang im Rahmen von Trockenbau- und Innenausbauarbeiten zeitweise auch brandempfindliche Bauteile zur Erhöhung der Feuerwiderstandsklasse mit asbesthaltigen Brandschutzplatten verkleidet hat. Die Dämmwolle bestand aber in aller Regel aus künstlichen Mineralfasern. Eine Einschätzung der kumulativen Asbestfaserstaubdosis liegt deutlich unterhalb von 2,5 Faserjahren. Es bleibt festzustellen, dass der Verstorbene abstrakt bis zum 31.03.1970 zeitweise der Einwirkung durch Asbestfaserstäube oberhalb des Grenzwertes von 250000 Fasern pro $\mathrm{m}^{3}$ ausgesetzt war."Asbestfaserjahre $<2,5$.

Radiologische Befunde: Januar 2007 Thorax-CT: „Tumoröse Raumforderung im rechten Lungenoberlappen mit Pleuraschwiele, keine asbestassoziierten Lungenveränderungen.“ 
Pathologie: Die Präparate (Lungenoberlappenresektat) wurden zunächst in einem auswärtigen Institut (Januar 2007) untersucht. Da zu diesem Zeitpunkt keine Berufskrankheit zur Diskussion stand, wurden ergänzende Untersuchungen (Eisenfärbung) oder histologische Untersuchungen zu einer asbestassoziierten Lungenfibrosierung und eine Lungenstaubanalyse nicht durchgeführt. Es wurde ein primärer bösartiger Lungentumor (Adenokarzinom) gesichert, die Pleura parietalis zeigte eine chronische Entzündung und eine Pleuraschwarte. Pleuraplaques wurden in dem resezierten Gewebe nicht belegt. Im Lungenparenchym fanden sich ein Emphysem und eine floride Bronchitis.

Erst Anfang 2009 wurde das Gewebe zur Untersuchung hinsichtlich asbestassoziierter Lungenveränderungen dem Mesotheliomregister übersandt.

Ergebnisse: Minimalasbestose (Grad I) ( $\bullet$ Abb. 3).

Lungenstaubanalyse: Bis zu 2000 Asbestkörper pro $\mathrm{cm}^{3}$ Lungengewebe. Bei den Asbestkörpern ( $\bullet$ Abb. 4) handelte es sich nach dem Ergebnis der Lungenstaubanalyse teils um sehr kleine Strukturen $(\bullet$ Abb. 5, $\odot$ Abb. 6, $\odot$ Abb. 7, $\odot$ Abb. 8), die eine intensive Suche im histologischen Präparat bei 400 -facher Vergrößerung notwendig machten.

Nach intensiver Suche konnten wiederholt, in Fibrosierungsarealen eingelagert, Asbestkörper nachgewiesen werden. Die Kriterien für eine Minimalasbestose sind erfüllt.

Pathologisch anatomische Bewertung: Kriterien für eine BK 4104 erfüllt.

\section{Diskussion}

Die Fallbeispiele 1 und 2 zeigen die Grenzen der radiologischen Diagnostik - konventionellen CT-Untersuchungen - bei der Bewertung asbestassoziierter Pleuraveränderungen auf. Durch die Obduktionsergebnisse wird eindrucksvoll bestätigt, dass sich Pleuraplaques einer konventionellen CT-Untersuchung mit einer Auflösungsgrenze von ca. $3 \mathrm{~mm}$ im Einzelfall entziehen können. Es handelt sich sicherlich um besondere Fallkonstellationen, aber die Möglichkeit, dass sich Pleuraplaques einer CT-Untersuchung entziehen, muss berücksichtigt werden.

Zu den Grenzen und Möglichkeiten der radiologischen Diagnostik wird in der entsprechenden Literatur ausgeführt, dass bei Röntgenthorax-Aufnahmen bis zu 60\% der im Rahmen einer Obduktion nachweisbaren Pleuraplaques übersehen werden und zu $20 \%$ falsch positive Befunde vorliegen [16]. CT-Untersuchungen weisen eine höhere Sensitivität und Spezifität auf: „a CT scan typically will show more pleural plaques and calcification, high-resolution CT (HRCT) is more sensitive“ [16-18]. Zur Sensivität und Spezifität $[17,18]$ beim Vergleich von CT und HRCT Untersuchungen wird festgestellt „high solution CT had a 97\% sensitivity and $100 \%$ specificity in the detection of pleural disease as a whole." Da eine Korrelation mit Obduktionsergebnissen bislang fehlt [17], bleibt es ungeklärt, ob eine Sensitivität und Spezifität von $100 \%$ beim Vergleich HRCT-Untersuchung versus Obduktion im Hinblick auf asbestassoziierte Pleuraveränderungen erreicht wird. Diese Fragestellung sollte in einer entsprechenden Studie untersucht werden. Da die HRCT-Untersuchung die sensitivste radiologische Methode zur Klärung asbestassoziierter Veränderungen darstellt, sollte diese als Standardmethode zur Bewertung von asbestassoziierten Veränderungen eingesetzt werden $[19,20]$. Bei der Bewertung einer CT-Befundung müssen die verwendeten Schichtdicken, Überlappung und Schichtabstände berücksichtigt werden $[18,20-22]$.

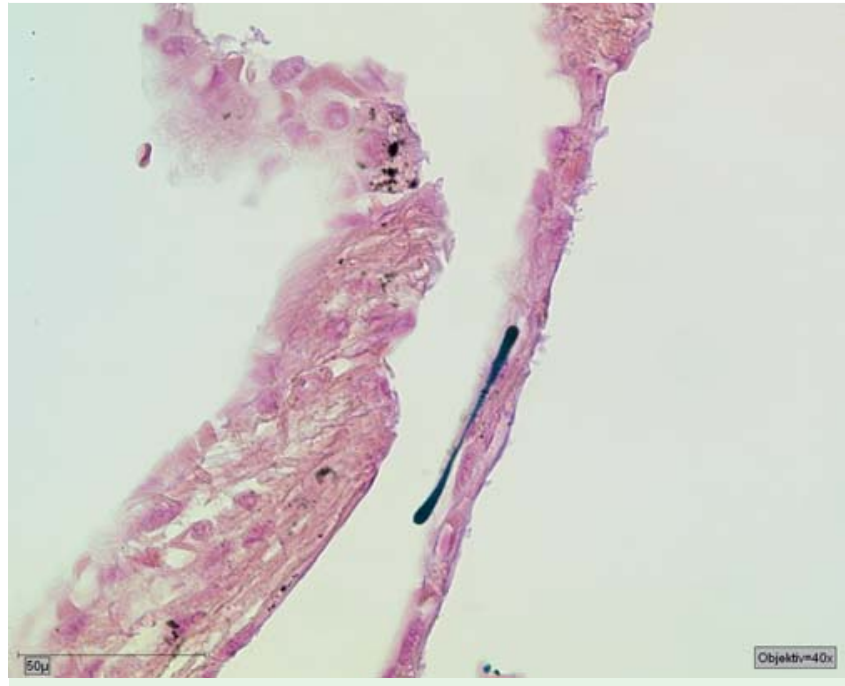

Abb. 3 Fall 3 Histologische Befunde eines Asbestkörpers in der Eisenfärbung bei 400 -facher Vergrößerung.

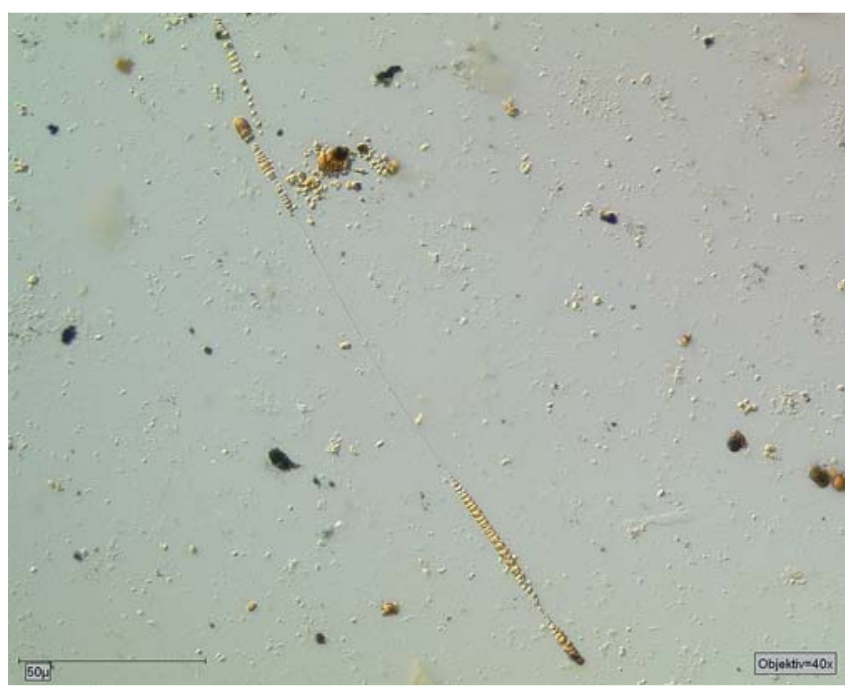

Abb. 4 Fall 3 Asbestkörper auf einem Filterpräparat bei 400-facher Vergrößerung (Differenzial-Interferenz-Kontrast).

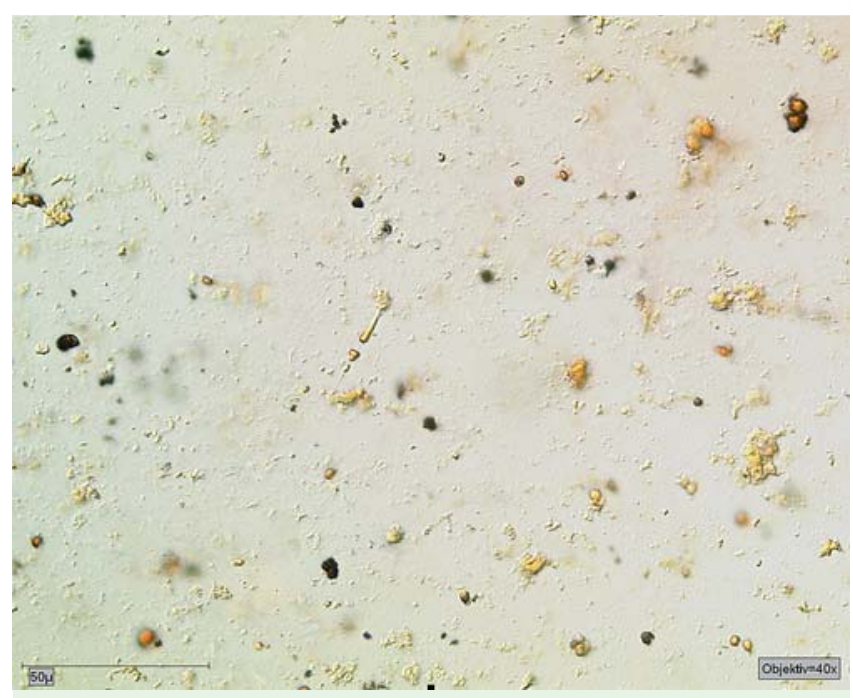

Abb. 5 Fall 3 Sehr kleiner Asbestkörper bei 400-facher Vergrößerung auf einem Filterpräparat (Differenzial-Interferenz-Kontrast). 


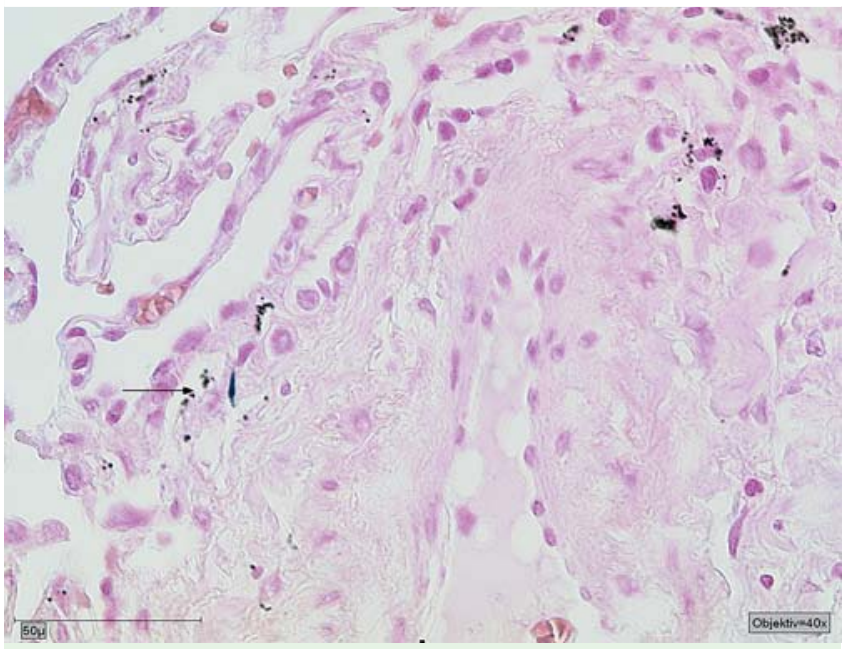

Abb. 6 Fall 3 Sehr kleiner Asbestkörper (Pfeil) in der Eisenfärbung bei 400facher Vergrößerung.

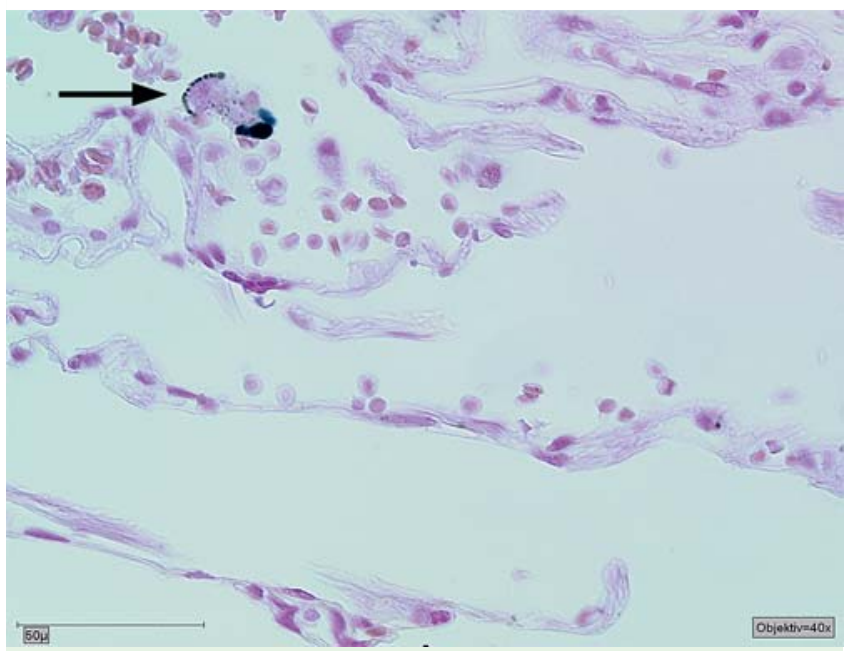

Abb. 7 Fall 3 Kleiner Asbestkörper (Pfeil) in der Eisenfärbung bei 400-facher Vergrößerung.

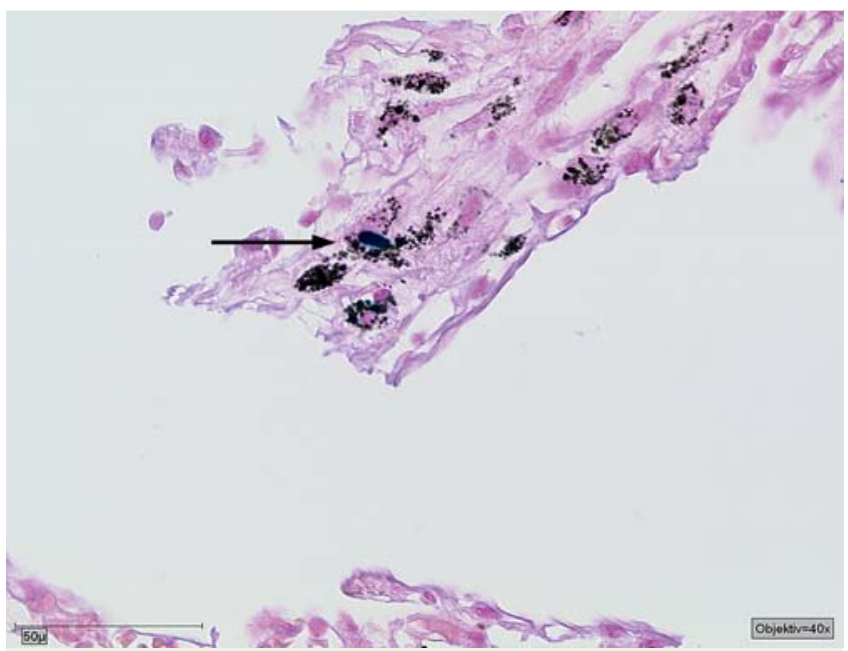

Abb. 8 Fall 3 Kleiner Asbestkörper (Pfeil) in der Eisenfärbung bei 400-facher Vergrößerung.
Fallbeispiel 3 zeigt, dass die kumulative Asbestfaserstaubdosis nicht immer mit der pulmonalen Asbestbelastung korreliert und nicht geeignet ist, auf das Vorliegen von asbestassoziierten Lungenfibrosierungen zurückzuschließen. Dies steht in guter Übereinstimmung mit den Ergebnissen einer größeren Studie ( $n=366$ ). Bei dieser Untersuchung erreichten $42,5 \%$ der Patienten mit histologisch gesicherten asbestassoziierten Lungenveränderungen (Minimalasbestosen Grad I und Asbestosen Grad II - IV) nicht $\geq 25$ Faserjahre [23]. Eine vergleichsweise niedrige kumulative Asbestfaserstaubdosis ist kein Ausschlusskriterium/Abschneidekriterium im Hinblick auf das Vorliegen einer asbestassoziierten Lungenfibrosierung. In diesem Zusammenhang ist auch eine HRCT-basierte Studie [24] von Interesse, in der mittels HRCT schon bei kumulativen Dosen von 1 Faserjahr Asbestosen gefunden wurden. Demzufolge ist das Kriterium der Faserjahre auch kein geeignetes Instrument für die Interpretation von Befunden mittels bildgebender Verfahren.

Im Deutschen Mesotheliomregister wurden wiederholt Lungengewebsproben untersucht, in denen nach der auswärtigen Routineuntersuchung - d.h. primär auf die Tumordiagnostik ausgerichteter Untersuchung - keine asbestassoziierte Lungenfibrosierung beschrieben wurde [25], jedoch nach gezielter histomorphologischer und ergänzender staubanalytischer Untersuchung unter Berücksichtigung der international und national anerkannten Kriterien [6,7] definitiv eine Minimalasbestose diagnostiziert wurde. Hieraus ist abzuleiten, dass der Nachweis von minimalen asbestassoziierten Lungenfibrosierungen nicht zwangsläufig im Rahmen der Routinediagnostik geleistet werden kann. Ohne den Befund der lichtmikroskopischen Lungenstaubanalyse ist die Diagnose einer Minimalasbestose - gerade vor dem Hintergrund, dass es sich um sehr kleine Asbestkörper handeln kann - deutlich erschwert. Entsprechende Studien stellen in diesem Zusammenhang fest; ,the optical examination of ABs obtained from the digested lung and observed lying on the membrane may be an easier method for the recognition and more reliable light microscopic indicator of the previous asbestos exposure" [4].

Die Untersuchung von auf Filtern angereicherten Lungenstäuben ist ein wertvolles Werkzeug und liefert neben der Asbestkörperkonzentration auch Informationen über Art und Größe der Strukturen, die im Lungengewebe zu suchen sind $[3,9,26]$. Zur Veranschaulichung der Asbestkörperanreicherung mittels der Lungenstaubanalytik kann die Überlegung dienen, dass bei einer Schnittdicke von ca. $5 \mu \mathrm{m}$ ein Gewebewürfel von $1 \mathrm{~cm}$ Kantenlänge 2000 histologische Schnittpräparate ergäbe [26]. Das heißt: Eine einzige Asbestkörperanreicherung aus einer Probe von $1 \mathrm{~cm}^{3}$ mit anschließender staubanalytischer Untersuchung ist vergleichbar mit der Auswertung von 2000 histologischen Schnittpräparaten.

Die Hypothese, dass Asbestkörper inaktive „Grabsteine“ [27] für Asbestfasern seien und diese somit keine Rolle mehr für die Genese asbestassoziierter Erkrankungen spielen, wird durch neue Untersuchungen widerlegt [28]. Asbestkörper weisen vielfach Stellen auf, an denen die Asbestfasern frei liegen. An diesen Stellen interagieren die Asbestfasern mit der Umgebung, ,isolated AB (asbestos bodies) were shown to be cytotoxic and activated free radical production from inflammatory cells" [28]. Der Nachweis von Asbestkörpern ist folglich nicht nur das entscheidende Merkmal zur Unterscheidung von Asbestosen gegenüber sonstigen Lungenfibrosen, sondern zeigt auch gleichzeitig die aktiv an dem pathogenen Prozess beteiligten Strukturen. Wenn eine Lungenfibrosierung unklarer Genese vorliegt und der lichtmikroskopische Nachweis von Asbestkörpern nicht gelingt, wird entspre- 
chend den Helsinki Kriterien [7] eine elektronenmikroskopische Faseranalytik notwendig. Diese Untersuchung erfolgt in einem Labor, das Referenzwerte für den Wertebereich von Asbestfaserkonzentrationen von so genannten Normallungen und für $\mathrm{Pa}$ tienten mit histologisch verifizierten Minimalasbestosen und Asbestosen ermittelt hat. Wenn die Anzahlkonzentrationen von Asbestfasern im Bereich von verifizierten Asbestosen des gleichen Labors liegen, wird dieses Ergebnis zur Unterscheidung von einer idiopathischen Lungenfibrose verwandt [7].

\section{Welche Auswirkungen sind bei Nichtanwendung aller zur Verfügung stehenden Untersuchungsverfahren zu befürchten? \\ $\nabla$}

Leider stellt die Dunkelziffer im Bereich der Berufskrankheiten eine Tatsache dar. Sowohl internationale Studien $[4,29,30]$ als auch die Erfahrungen im Deutschen Mesotheliomregister sind hier eindeutig. Die Untersuchungen [4] weisen auf die enorme Tragweite von pathologisch-anatomischen und staubanalytischen Untersuchungen hin. Durch Extrapolation gelangte man zu einer Häufigkeit von 2000 Fällen allein von asbestassoziierten Bronchialkarzinomen in Italien pro Jahr, davon anerkannt wurden in Italien in diesem Zeitraum aber nur 281 Berufkrankheiten (from all occupational causes) [4]. Bedauerlicherweise werden nicht immer entsprechende Analysen durchgeführt, selbst dann nicht, wenn geeignetes Untersuchungsgut zur Verfügung steht.

\section{Fazit für die Praxis}

\section{$\nabla$}

Eine pathologisch-anatomische Untersuchung unter Zuhilfenahme der Lungenstaubanalytik ist - bei fehlendem Nachweis von pleuralen Brückenbefunden und/oder Nichterreichen der $\geq 25$ Asbestfaserjahre - für die Bewertung von asbestassoziierten Lungenerkrankungen unverzichtbar. Für die radiologische Beurteilung ist eine HRCT-Befundung das Mittel der Wahl. Die Nichtausschöpfung aller Beweismittel, z.B. durch Verzicht auf pathologisch-anatomische und lungenstaubanalytische Untersuchungen, führt dazu, dass ein nicht unerheblicher Anteil von Berufskrankheiten nach Ziffer 4104 nicht entschädigt wird. Eine entsprechende Studie sollte diesen Sachverhalt in Deutschland eingehender untersuchen. Die definitive Ablehnung einer Berufskrankheit nach Ziffer 4104 ohne die Untersuchung von Lungengewebe ist somit nicht gerechtfertigt. Da invasive Eingriffe ausschließlich zur Gewinnung von Gewebe für staubanalytische Untersuchungen nicht zulässig sind [9], muss die Möglichkeit einer Obduktion geprüft werden.

\section{Interessenkonflikte}

$\nabla$

Bei den Autoren besteht kein Interessenkonflikt im Sinne der Richtlinien des International Committee of Medical Journal Editors.

\section{Literatur}

1 Müller K, Krismann M. Asbestassoziierte Erkrankungen. Deutsch Ärztebl 1995; 93: A538-A543

2 Müller K, Wiethege T. Pathology, classification and staging of malignant lung tumors. Radiologie 2004; 44: 415-426
3 Manning C, Vallyathan V, Mossmann B. Diseases caused by asbestos: mechanisms of injury and disease development. Int Immunoph 2002; 2: $191-200$

4 Mollo F, Magnani C, Bo P et al. The attribution of lung cancer to asbestos exposure. Am J Clin Pathol 2002; 117: 90 - 95

5 Raithel H, Kraus T, Hering $\mathrm{K}$ et al. Asbestbedingte Berufskrankheiten. Deutsch Ärztebl 1996; 93: A685-A693

6 Heitz P. Neue Definition der Minimalasbestose. Deutsch Ärzteblatt 1997; 94: A975

7 Henderson D, Rantanen J, Barnhart S et al. Helsinki criteria Asbestos, asbestosis and cancer. The Helsinki criteria for diagnosis and attribution. Scand J Work Enviro Health 1997; 23: 311 - 316

8 Guidotti T, Miller A, Christiani D et al. American thoracic society documents: Diagnosis and initial management of non-malignant diseases related to asbestos. Am J Repir Crit Care Med 2004; 170: 691 - 715

9 Müller K, Fischer M. 10 Jahre Mesotheliomregister. Kompass 1997; 7 8: $308-312$

10 Eitner F, Otto H. Zur Dignität von Asbestkörperzählungen im Lungengewebe. Arbeitsmed Sozialmed Präventivmed 1984; 19: 1 -5

11 Brockmann M. Forschungsbericht Pleuramesotheliom: Malignes diffuses Pleuramesotheliom - Heterogenität, Differentialdiagnose und Histogenese. Sankt Augustin: HVGB, 1992

12 Wächli P, Rajower I, Christen B et al. Ferruginous Bodies im Lungenstaub als Indikatoren für Asbestschäden. Pathologe 1987; 8: 346-350

13 Brockmann M. Asbestassoziierte Lungen- und Pleuraerkrankungen pathologische Anatomie. Pneumologie 1991; 45: 422-428

14 DeVuyst P, Karjalainen A, Dumortier P et al. Guidelines for mineral fibre analysis i biological samples : Report of the ERS Working group. Eur Resp J 1998; 11: 1416 - 1426

15 Roggli $V$, Pratt $P$. Numbers of asbestos bodies on iron stained tissue sections in relation to asbestos body count in lung tissue digests. Hum Path 1983; 14: 355-361

16 Clarke C, Mowat F, Kelsh M et al. Pleural plaques: A Review of diagnostic issues and possible nonasbestos factors. Arch of Envir Occup Health 2006; 61: 183-192

17 Friedmann A, Fiel S, Fisher $M$ et al. Asbestos related pleural disease and asbestosis: A comparison of CT and chest radiography. Am J Rad 1988; 150: $269-275$

18 Roach H, Davies G, Attanoos R et al. Asbestos: When the dust settles an imaging review of asbestos related diseases. Radiographics 2002; 22: $167-184$

19 Hering K, Tuengerthal S, Kraus T. Standardisierte CT/HRCT Klassifikation der Bundesrepublik Deutschland für arbeits- und umweltbedingte Thoraxerkrankungen. Radiologie 2004; 44: 500-511

20 Kraus T, Raithel H, Lehnert G. Computed assisted classification system for chest X-ray and computed findings in occupational disease. Int Arch Occup Enviro Health 1997; 69: 482 - 486

21 Akira M, Yamamoto S, Inoue Yet al. High resolution CT of asbestosis and idiopathic pulmonary fibrosis. Am J Respir 2003; 181: 163 - 169

22 Paris C, Thierry S, Brochard P et al. Pleura plaques and asbestos: Dose and time response relationships based on HRCT data. Eur Respir J 2009; in press

23 Fischer M, Günther S, Müller K. Faserjahre, Asbestbelastung der Lunge und Asbestosen. Pneumologie 2000; 54: 155 - 159

24 Arhelger $R$, Mayer $P$, Hering $K$ et al. Korrelation der Streuungsgrade einer Lungenasbestose nach ILO und HRCT mit der kumulativen Asbestfaserstaub-Dosis. Arbeits Sozialmed Umwelt 2009; 44: 183

25 Tannapfel A. Deutsches Mesotheliomregister - Jahresbericht 2007. Kompass 2008; 7/8: $22-27$

26 Otto H, Bohlig $H$. Morphologie und Röntgenologie der Asbestose. Radiologe 1985; 25: 9-21

27 Woitowitz H. Kausalitätsprobleme bei der Begutachtung asbestverursachter Tumoren. 1986; Vortrag Tagung „Asbest Spätschäden“ Bad Nauheim. 3 September 1986

28 Borelli $V$, Brochetta C, Maur M et al. A procedure for the Isolation of asbestos bodies from lung tissue. J. Toxicol. Enviro Health 2007; 70: $1232-1240$

29 Baroetavena $M$, Teschke $K$, Bates $D$. Unrecognized asbestos induced diseases. Am J Ind Med 1996; 29: 183-185

30 Kishimoto T, Ohnishi K, Saito Y. Clinical study of asbestos related lung cancer. Ind Health 2003; 41: 94-100 